2. Khan T, Joseph B. Controversies in the management of congenital pseudarthrosis of the tibia and fibula. Bone Joint J. 2013;95-B(8):1027-34.

doi: 10.1302/0301-620X.95B8.31434.

3. Pannier S. Congenital pseudarthrosis of the tibia. Orthop Traumatol Surg Res. 2011;97(7):750-61.

doi: 10.1016/j.otsr.2011.09.001.
4. Cho TJ, Choi IH, Lee KS, et al. Proximal tibial lengthening by distraction osteogenesis in congenital pseudarthrosis of the tibia. J Pediatr Orthop. 2007;27(8):915-20. doi: 10.1097/bpo.0b013e31815a6058.

\title{
FUNCTIONAL STATE OF BLOOD CIRCULATION AND NEUROMUSCULAR SYSTEM OF THE LOWER LIMB OF PATIENTS WITH CONGENITAL PSEUDARTHROSIS OF THE TIBIA AFTER CONSOLIDATION OF THE NONUNION
}

\author{
Pozdeev A.P. ${ }^{1}$, Zakharyan E.A. ${ }^{2}$, Zubairov T.F. ${ }^{1}$, Nikityuk I.E. ${ }^{1}$ \\ ${ }^{1}$ The Turner Scientific and Research Institute for Children's Orthopedics, Saint-Petersburg, Russian Federation \\ ${ }^{2}$ North-Western State Medical University n. a. I.I. Mechnikov, Saint-Petersburg, Russian Federation
}

The aim of this study was to evaluate the clinical and functional state of the neuromuscular system and the blood supply to the lower limbs of children with congenital pseudarthrosis of the tibia (CPT) after consolidation. Material and Methods. A total of 100 patients with CPT were analyzed. We performed a clinical examination of patients, panoramic X-ray of the lower extremities, electroneuromyogram, and reovasography. Results and Conclusions. The primary complaints of patients with CPT after the consolidation of the non-union were lameness, deformations of lower extremities, and pain in the local joints. The electromyoneuromyogram data of the lower limbs of patients with CPT exhibited a decrease of the contractility of the muscles of the lower limbs, and neuropathy of the peroneal nerves of both lower limbs. The reovasography data of the lower limbs of patients with CPT displayed improvement in blood circulation in the lower extremities after the consolidation of the tibia. These data promote the current methods of treatment of patients with CPT; however, the temperature, degree of limb lengthening, and deformity correction should be considered in the future.

Keywords: congenital pseudarthrosis of the tibia, correction deformity, neuropathy, shortening.
Поздеев Александр Павлович - д. м. н., профессор, главный научный сотрудник отделения костной патологии ФГБУ «НИДОИ им. Г.И. Турнера» Минздрава России. E-mail: professorpozdeev@mail.ru.

Захарьян Екатерина Анатольевна - аспирант кафедры детской травматологии и ортопедии ГБОУ ВПО «СЗГМУ им. И.И. Мечникова» Минздрава России. E-mail: zax-2008@mail.ru.

Зубаиров Тимур Фаизович - к. м. н., научный сотрудник отделения костной патологии ФГБУ «НИДОИ им. Г.И. Турнера» Минздрава России.

Никитюк Игорь Евгеньевич - к. м. н., ведущий научный сотрудник лаборатории физиологических и биомеханических исследований ФГБУ «НИДОИ им. Г.И. Турнера» Минздрава России. E-mail: femtotech@mail.ru.
Pozdeev Aleksander Pavlovich - MD, PhD, professor, chief research associate of the department of bone pathology. The Turner Scientific and Research Institute for Children's Orthopedics. E-mail: professorpozdeev@mail.ru.

Zakharyan Ekaterina Anatolievna - MD, PhD student of the chair of pediatric traumatology and orthopedics. NorthWestern State Medical University n. a. I.I. Mechnikov. E-mail: zax-2008@mail.ru.

Zubairov Timur Faizovich - MD, PhD, research associate of the department of bone pathology. The Turner Scientific and Research Institute for Children's Orthopedics.

Nikityuk Igor Evgenievich - $\mathrm{MD}, \mathrm{PhD}$, leading research associate of the laboratory of physiological and biomechanical research. The Turner Scientific and Research Institute for Children's Orthopedics. E-mail: femtotech@ mail.ru. 


\title{
АНААИЗ ВАИЯНИЯ РАЗАИЧНЫХ ФАКТОРОВ НА АИНАМИКУ НЕВРОАОГИЧЕСКИХ НАРУШЕНИЙ У АЕТЕЙ С ПОЗВОНОЧНО-СПИННОМОЗГОВОЙ ТРАВМОЙ
}

\author{
(C) Баиндурашвили А.Г., Солохина И.Ю., Кокушин Д.Н., Белянчиков С.М. \\ ФГБУ «НИДОИ им. Г.И. Турнера» Минздрава России, Санкт-Петербург
}

\begin{abstract}
Актуальность. Изучение влияния различных факторов на динамику восстановления неврологических нарушений у детей с позвоночно-спинномозговой травмой является важной и актуальной проблемой. Основными причинами повреждений грудного и поясничного отделов позвоночника у детей являются дорожно-транспортные происшествия и кататравмы. Возрастные анатомо-физиологические особенности позвоночника и спинного мозга у детей оказывают существенное влияние на характер повреждения спинного мозга, клинические проявления травмы, а следовательно, и на выбор лечебной тактики. Величина деформации позвоночного канала на уровне поврежденного сегмента прямо пропорционально влияет на выраженность неврологических нарушений. На характер неврологических нарушений и динамику восстановления двигательных функций существенное влияние оказывают сроки оперативного лечения, прошедшие от момента травмы.

Цель. Оценить влияние различных факторов у пациентов детского возраста с осложненными повреждениями позвоночника на грудном и грудопоясничном уровне на динамику восстановления неврологических нарушений.

Материалы и методы. Проведен анализ результатов хирургического лечения 36 детей (24 мальчика и 12 девочек) в возрасте от 3 до 17 лет с повреждением позвоночника и спинного мозга в грудном отделе позвоночника и области грудопоясничного перехода, сопровождающимся неврологическим дефицитом в виде центральных или периферических парезов и параличей. Всем пациентам выполнено хирургическое вмешательство в зависимости от варианта и уровня повреждений. В ходе исследования использовали клинический (подробный неврологический осмотр), рентгенологический, КТ-, МРТ-методы диагностики. Результаты. Проведенное исследование выявило, что наиболее тяжелые повреждения с точки зрения неврологических нарушений у детей с позвоночно-спинномозговой травмой возникают при локализации переломов в грудном отделе позвоночника. Характер неврологических изменений зависит от уровня повреждения позвоночного столба и коррелирует с величиной стеноза позвоночного канала. Операция, выполненная в первые часы от момента травмы, приводит к более быстрому и полноценному восстановлению неврологического дефицита.

Заключение. Таким образом, в ходе исследования установлено, что влияние на восстановление неврологических нарушений у детей с позвоночно-спинномозговой травмой оказывают многих факторы: сроки хирургического вмешательства, локализация повреждения, стеноз позвоночного канала, характер поражения спинного мозга и его элементов.
\end{abstract}

Ключевые слова: травма позвоночника и спинного мозга у детей, позвоночно-спинномозговая травма, шкала ASIA, хирургия позвоночника.

\section{Введение}

Согласно данным отечественной литературы частота встречаемости всех повреждений позвоночного столба у детей в общей структуре травм опорно-двигательного аппарата составляет от 1 до $10 \%$ с разбросом от 1,9 до 19,9 случая на миллион детского населения [1]. Статистические данные детских стационаров Санкт-Петербурга за 2012-2013 годы указывают, что ежегодно от 900 до 1100 детей получают переломы позвоночника различной локализации, из них от 2 до 3 \% приходится на долю нестабильных и осложненных повреждений позвоночного столба [2]. Сочетанное повреждение позвоночника и спинного мозга у детей составляет 2-5 \% от общего числа тяжелых травм позвоночного столба. Хотя, по мнению других авторов, переломы позвоночника у пациентов детского возраста в 20-34,5 \% наблюдений сопровождаются тем или иным вариантом повреждения спинного мозга, что опровергает точку зрения об исключительной редкости осложненных переломов позвоночника у детей [3]. 
Неврологическая симптоматика, возникающая при позвоночно-спинномозговой травме в грудном и поясничном отделах, и дальнейшая ее динамика очень разнообразны и зависят от многих факторов: характера перелома позвоночника, величины стеноза позвоночного канала, тяжести повреждения самого спинного мозга и его корешков (сотрясение, ушиб, сдавление, гематомиелия), а также сроков оперативного лечения. Взаимосвязь этих факторов напрямую влияет на процесс восстановления неврологических нарушений после выполненного хирургического вмешательства.

Нарушение функции проводящих путей спинного мозга в грудном отделе позвоночника, зоне поясничного утолщения спинного мозга, конского хвоста и спинномозговых корешков наблюдается в результате непосредственного воздействия повреждающей силы в момент травмы, компрессии костными отломками поврежденных тел позвонков и фрагментами диска, смещенными в позвоночный канал. Кроме того, присоединение в последующем сосудистых нарушений также оказывает влияние на формирование и развитие неврологического дефицита [4].

По данным литературных источников, наиболее тяжелые неврологические нарушения имеют пострадавшие с локализацией травмы позвоночника в грудном отделе, что объясняется анатомическими особенностями строения данного сегмента позвоночного столба [4]. Отличительной особенностью этого отдела позвоночника является узость позвоночного канала и ограниченное (щелевидное) пространство между его стенками и дуральным мешком. В связи с этим даже относительно небольшая компрессия костными отломками либо веществом межпозвоночного диска может привести к выраженному неврологическому дефициту.

Величина деформации позвоночного канала на уровне поврежденного сегмента прямо пропорционально влияет на выраженность неврологических нарушений. Так, при кифотической деформации позвоночника более $20^{\circ}$ и горизонтальном смещении костных отломков тел позвонков не менее 6 мм при переломовывихах отмечаются тяжелые неврологические расстройства в виде полного нарушения проводимости спинного мозга [5].

На характер неврологических нарушений и динамику восстановления двигательных функций существенное влияние оказывают сроки оперативного лечения, прошедшие от момента травмы. Ряд авторов считают, что хирургическое вмешательство в ранние сроки от момента повреждения не влияет на темпы восстановления неврологического дефицита, а даже, наоборот, может приводить к ухудшению состояния. Кроме того, отсутствует единое мнение и по поводу определения временных границ ранних сроков операции. T. Asawma et al. считают ранними сроками хирургического вмешательства первые 4 недели после травмы[6], J. Farmer et al. - первые 5 дней [7], A. Vaccaro и S. Mirsa - первые трое суток (72 часа) [8-9], a F. Wagner и B. Chehrazi первые 8 часов от момента повреждения [10].

A. Vaccaro et al. при сравнении результатов хирургического лечения пациентов с травмой шейного отдела позвоночника, оперированных в течение первых 72 часов от момента травмы, и пациентов, оперированных позднее 5 дней от момента повреждения, не выявили разницы в неврологическом исходе в обеих группах [8]. F. Wagner и B. Chehrazi для того, чтобы оценить влияние сохраняющейся компрессии спинного мозга на восстановление неврологических функций после травмы позвоночника и спинного мозга, рассмотрели 44 случая травмы на уровне С3-С7 позвонков [10]. Они оценивали характер выраженности неврологических нарушений с величиной стеноза позвоночного канала и сроками хирургического вмешательства от момента травмы. Первой группе больных декомпрессию и стабилизацию поврежденного сегмента позвоночника осуществляли в первые 8 часов от момента травмы, второй - проводили хирургическое лечение в том же объеме на протяжении 48 часов. Результаты оценивали сразу после операции и через год после вмешательства. Неврологический дефицит, который был у пациентов при поступлении, полностью коррелировался с величиной стеноза позвоночного канала. Однако существенной разницы в динамике регресса неврологических расстройств в обеих группах исследования отмечено не было. Авторы приходят к выводу, что первичная травма спинного мозга и позвоночника остается главным фактором, который определяет неврологический исход пациента. M. Fehlings et al. считают, что нет общепринятых стандартов относительно роли и выбора сроков хирургической декомпрессии, но одновременно с этим отмечают, что раннее оперативное вмешательство (до 24 ч) должно быть выполнено тем пациентам, у которых имеется выраженная неврологическая симптоматика [11].

H. Bohlman и P. Anderson представили peзультаты анализа 58 операций в позднем периоде (от 1 месяца до 9 лет от момента повреждения) у пациентов с позвоночно-спинномозговой травмой. В группе исследованных 29 пациентов после операции начали ходить, 6 больных, которые ходили до вмешательства, стали передвигаться значительно лучше [12]. Только у 9 из них не отмечалось улучшения в неврологическом статусе. Однако авторы не указывают, в какие сроки после операции 
появилось улучшение и делают оговорку, что, возможно, оно бы возникло и без вмешательства.

А.Г. Аганесов и др. считают, что эффективность хирургического лечения осложненной травмы позвоночника в остром и раннем периодах зависит прежде всего от срока проведения оперативного вмешательства, скорейшей декомпрессии и стабилизации поврежденного позвоночно-двигательного сегмента [1].

Ишемия спинного мозга является непременным компонентом его травматического повреждения. Сдавление его сосудов без травматического повреждения клеток приводит к ишемическому некрозу, развитию воспалительной реакции и запускает апоптоз. Уже через 180 минут компрессии сосудов мозга наступают полностью необратимые изменения нейронов [13]. По данным А.А. Луцика, при компрессии спинного мозга, как правило, сдавливаются его магистральные сосуды, особенно передняя спинальная артерия и отходящие от нее центральные артерии спинного мозга [14]. Это диктует необходимость осуществлять его декомпрессию максимально быстро, иначе в спинном мозге могут наступить необратимые изменения. Количественный анализ острой аксональной патологии при экспериментальной контузии спинного мозга показал общую тенденцию в его повреждении: в течение 15 минут повреждается серое вещество, в течение 4 часов - белое. Медленное развитие повреждения белого вещества дает терапевтическое «окно» для воздействия на патологический процесс [15].

Целью исследования явилась оценка влияния различных факторов на динамику неврологиче- ских нарушений у детей с позвоночно-спинномозговой травмой в грудном отделе позвоночника и в области грудопоясничного перехода.

\section{Материалы и методы}

Проведен анализ результатов хирургического лечения 36 детей (24 мальчика и 12 девочек) в возрасте от 3 до 17 лет с повреждениями позвоночника и спинного мозга в грудном отделе и области грудопоясничного перехода, сопровождающимися неврологическими нарушениями в виде центральных или периферических парезов и параличей. Пациенты старше 11 лет составили 77,8 \% от общего числа всех пострадавших (табл. 1).

Основными причинами возникновения позвоночно-спинномозговой травмы у детей в 19 наблюдениях (52,8 \%) являлось дорожно-транспортное происшествие, в 17 (47,2 \%) - кататравма.

У всех пациентов в исследовании использовали клинический метод (подробный неврологический осмотр пациента), рентгенографию поврежденного отдела позвоночника, компьютерную и магнитно-резонансную томографию. Оценку стеноза позвоночного канала выполняли по аксиальным и сагиттальным сканам компьютерных томограмм с математическим обсчетом полученных данных. С целью максимальной стандартизации результатов клинического исследования использовали шкалу ASIA, разработанную Американской ассоциацией спинальной травмы, включающей в себя балльную оценку мышечной силы и чувствительности (тактильная и болевая) [16].

Таблица 1

Распределение пациентов с осложненными повреждениями позвоночника в грудном отделе и области грудопоясничного перехода по возрасту

\begin{tabular}{|l|c|c|c|c|}
\hline \multicolumn{1}{|c|}{ Возраст } & от 3 до 7 лет & от 7 до 11 лет & от 11 до 17 лет & Всего \\
\hline Мальчики & 4 & 4 & 16 & $24(66,7 \%)$ \\
\hline Девочки & - & - & 12 & $12(33,3 \%)$ \\
\hline Всего & $4(11,1 \%)$ & $4(11,1 \%)$ & $28(77,8 \%)$ & $36(100 \%)$ \\
\hline
\end{tabular}

таблица 2

Распределение пациентов с осложненными повреждениями позвоночника в зависимости от типа неврологических нарушений и ^окализации повреждения

\begin{tabular}{|c|c|c|c|c|c|c|}
\hline \multirow{2}{*}{ Группы } & \multirow{2}{*}{ Локализация повреждения } & \multicolumn{4}{|c|}{ Тип неврологических нарушений по шкале ASIA } & \multirow{2}{*}{ Всего } \\
\hline & & A & $\mathrm{B}$ & $\mathrm{C}$ & $\mathrm{D}$ & \\
\hline I & $\begin{array}{l}\text { Грудной } \\
\text { Грудопоясничный }\end{array}$ & $\begin{array}{l}2 \\
-\end{array}$ & $\overline{6}$ & $\begin{array}{l}- \\
-\end{array}$ & $\begin{array}{l}- \\
-\end{array}$ & 8 \\
\hline II & $\begin{array}{l}\text { Грудной } \\
\text { Грудопоясничный }\end{array}$ & - & - & $\begin{array}{l}- \\
- \\
\end{array}$ & $\overline{2}$ & 4 \\
\hline III & $\begin{array}{l}\text { Грудной } \\
\text { Грудопоясничный }\end{array}$ & $\begin{array}{l}2 \\
- \\
\end{array}$ & - & $\overline{4}$ & $\begin{array}{l}- \\
- \\
\end{array}$ & 8 \\
\hline IV & $\begin{array}{l}\text { Грудной } \\
\text { Грудопоясничный }\end{array}$ & $\begin{array}{l}8 \\
2\end{array}$ & $\begin{array}{l}4 \\
2\end{array}$ & $\begin{array}{l}- \\
-\end{array}$ & $\begin{array}{l}- \\
-\end{array}$ & 16 \\
\hline Всего & $\begin{array}{l}\text { Грудной } \\
\text { Грудопоясничный }\end{array}$ & $\begin{array}{c}12 \\
4\end{array}$ & $\begin{array}{c}4 \\
10\end{array}$ & $\overline{4}$ & $\overline{2}$ & $\begin{array}{l}16 \\
20\end{array}$ \\
\hline
\end{tabular}


Согласно данным, представленным в табл. 2, установлено, что из 36 пациентов 16 больных имели исходный уровень неврологических нарушений - тип А, 14 травмированных - тип В, 4 детей - тип С и у 2 пациентов неврологический дефицит соответствовал типу D по шкале ASIA. Также необходимо подчеркнуть, что синдром полного нарушения проведения спинного мозга имели 12 пациентов с уровнем позвоночно-спинномозговой травмы в грудном отделе и 4 детей с локализацией повреждения в зоне грудопоясничного перехода.

Для оценки характера костных переломов позвоночника использовали классификацию F. Magerl et al. [17]. Переломы типа А3 встречались у 18 (50\%) пациентов, типа В - у двух $(5,6 \%)$ и типа С - у $16(44,4 \%)$ детей. По локализации повреждения позвоночника распределялись следующим образом: грудной отдел позвоночника $-16(44,4 \%)$ пострадавших, грудопоясничный переход (Th10-L2) - 20 (55,6 \%) пациентов.

Всем пациентам выполнено хирургическое вмешательство с учетом варианта повреждения позвоночника из комбинированного или дорсального доступа в объеме фиксации поврежденного позвоночно-двигательного сегмента и декомпрессии структур спинного мозга и его элементов [2-4]. Больным с повреждениями типа А и В по классификации F. Magerl оперативное вмешательство выполняли одномоментно из двух доступов. Из дорсального подхода осуществляли заднюю непрямую репозицию и стабилизацию поврежденного позвоночно-двигательного сегмента с задним локальным спондилодезом. Из переднебокового торакального, люмботомического или торакофренолюмботомического доступа выполняли декомпрессию спинного мозга и его элементов путем удаления костных отломков тела сломанного позвонка, восстановление анатомии позвоночного канала, реконструкцию передней и средней колонн на этом уровне pyramesh в сочетании с костной пластикой. При переломах типа С весь объем хирургического вмешательства осуществляли только из заднего доступа. Целью операции являлось устранение всех видов дислокационных смещений позвонков в поврежденном позвоночно-двигательном сегменте при помощи многоопорной металлоконструкции, стабилизация в физиологически правильной позиции травмированного сегмента позвоночника как во фронтальной, так и в сагиттальной плоскости после осуществления декомпрессии спинного мозга и ревизии позвоночного канала. Завершалось вмешательство формированием корпородеза и заднего локального спондилодеза.
С момента поступления в стационар всем пациентам с неврологическими нарушениями проводили гормональную терапию по схеме, рекомендуемой протоколом NASCIS-I: болюсное введение 30 мг/кг в первые 6 часов, затем 5,4 мг/кг в час из расчета по метилпреднизолону в течение 23 часов.

После хирургического вмешательства пациенты продолжали получать начатую во время операции гормональную терапию в дозировке 5,4 мг/кг в час в течение 48 часов. В ближайшем послеоперационном периоде при условии стабильной гемодинамики:

1) проводили дегидратационную терапию (лазикс 2-3 мг/кг в сутки на 3-4 приема, фуросемид 20-60 мг в сутки);

2) проводили гемостатическую терапию (дицинон 3-7 мг/кг 4 раза в сутки);

3) со 2-3-го дня после операции назначали сосудистые препараты (нимотоп 5-10 мл/ч в течение 10-14 дней);

4) назначали витамины группы В (нейромультивит).

Терапию сосудистыми препаратами проводили при отсутствии угрозы продолжающегося кровотечения и стабильной гемодинамики у пациента.

Кроме этого всем больным осуществляли интенсивное восстановительное лечение, включающее пассивную лечебную физкультуру (по нескольку раз в день), массаж конечностей, специальные укладки, двигательную реабилитацию с использованием механотерапии и роботизированных систем.

Средний срок стационарного лечения пациентов с осложненными повреждениями позвоночника в грудном отделе позвоночника и области грудопоясничного перехода составил 24 дня. В дальнейшем детей переводили для продолжения восстановительного лечения в реабилитационные центры. После выписки из стационара пострадавшие продолжали медикаментозную терапию.

В ходе исследования оценивали влияние различных факторов у пациентов детского возраста с позвоночно-спинномозговой травмой на динамику неврологических нарушений после хирургического лечения. Многофакторную оценку у пациентов с осложненными повреждениями позвоночника в грудном отделе позвоночника и зоне грудопоясничного перехода проводили по следующим критериям:

- выраженность неврологических нарушений в зависимости от уровня повреждения;

- сроки оперативного вмешательства, прошедшие от момента травмы;

- динамика неврологических нарушений после операции (по шкале ASIA). 
Мониторинг динамики неврологического статуса осуществляли в ближайшие сроки после операции (ежедневно в течение недели, затем 1 раз в 2-3 дня весь период пребывания пациента в стационаре). В дальнейшем пациенты осматривались каждые полгода с обязательным занесением данных неврологического осмотра в протокол спинальной травмы. Отдаленный результат лечения прослежен в период до 5 лет у всех пациентов.

\section{Результаты}

В ходе исследование отмечено, что при локализации переломов позвоночника в грудном отделе возникают более тяжелые повреждения спинного мозга с точки зрения неврологических нарушений. У 16 пациентов с локализацией травмы в грудном сегменте позвоночного столба наблюдался самый тяжелый тип неврологического дефицита - A и В по шкале ASIA, при этом у 12 из них отмечался тип А и только у $4-$ тип В.
У 20 больных с локализацией повреждения позвоночника в зоне грудопоясничного перехода имели место все варианты неврологических нарушений от типа А до типа $\mathrm{D}$, при этом 50 \% (10 детей) составили пациенты с типом неврологического дефицита В по шкале ASIA.

Сроки от момента повреждения позвоночника и спинного мозга до времени проведения хирургического вмешательства у пациентов с позвоночноспинномозговой травмой варьировали от нескольких часов до 18 месяцев. В зависимости от этого факта все пострадавшие были разделены на 4 группы. Согласно данным, представленным в табл. 3, в первые 6-12 часов от момента травмы прооперировано 8 детей, в остром периоде вертебро-медулярной травмы (от 12 часов до 3 дней) - 4 пациента. Остальные пациенты получили хирургическое лечение в раннем периоде от момента повреждения (от 3 дней до 2 недель) -8 человек, в промежуточном и в позднем периодах (от 14 дней до 18 месяцев) 16 детей.

Таблица 3

Распределение пациентов по характеру повреждения позвоночника и срокам хирургического лечения

\begin{tabular}{|c|c|c|c|c|c|c|c|}
\hline \multirow{3}{*}{$\begin{array}{c}\text { Характер повреждения } \\
\text { Сроки хирургического } \\
\text { лечения }\end{array}$} & \multicolumn{2}{|c|}{ Тип А3 } & \multicolumn{2}{|c|}{ Тип В } & \multicolumn{2}{|c|}{ Тип C } & \multirow{3}{*}{ Всего } \\
\hline & \multicolumn{6}{|c|}{ Локализация повреждения } & \\
\hline & Грудной & $\begin{array}{c}\text { Грудопояс- } \\
\text { ничный }\end{array}$ & Грудной & $\begin{array}{c}\text { Грудопояс- } \\
\text { ничный }\end{array}$ & Грудной & $\begin{array}{c}\text { Грудопояс- } \\
\text { ничный }\end{array}$ & \\
\hline $\begin{array}{l}\text { Первые 6-12 часов от мо- } \\
\text { мента травмы (I группа) }\end{array}$ & - & 6 & - & - & 2 & - & 8 \\
\hline $\begin{array}{l}\text { От } 12 \text { часов до } 3 \text { дней } \\
\text { (II группа) }\end{array}$ & - & 2 & - & - & - & 2 & 4 \\
\hline $\begin{array}{l}\text { От } 3 \text { дней до } 14 \text { дней } \\
\text { (III группа) }\end{array}$ & - & 4 & - & - & 2 & 2 & 8 \\
\hline Более 14 дней (IV группа) & 4 & 2 & 2 & - & 6 & 2 & 16 \\
\hline Итого & 4 & 14 & 2 & - & 10 & 6 & 36 \\
\hline
\end{tabular}

Таблица 4

Показатели стеноза позвоночного канала до операции и в раннем послеоперационном периоде

\begin{tabular}{|c|c|c|c|}
\hline \multirow{2}{*}{ Группа } & \multirow{2}{*}{ Локализация повреждения } & \multicolumn{2}{|c|}{ Стеноз позвоночного канала в \% } \\
\hline & & До операции & После операции \\
\hline I & $\begin{array}{l}\text { Грудной } \\
\text { Грудопоясничный }\end{array}$ & $\begin{array}{c}\text { от } 90 \text { до } 97 \\
93,5 \pm 3,5 \\
\text { от } 73 \text { до } 94 \\
83,7 \pm 6,7\end{array}$ & $\begin{array}{l}0 \\
0\end{array}$ \\
\hline II & Грудопоясничный & $\begin{array}{c}\text { от } 42 \text { до } 88 \\
65,0 \pm 22\end{array}$ & 0 \\
\hline III & $\begin{array}{l}\text { Грудной } \\
\text { Грудопоясничный }\end{array}$ & $\begin{array}{c}\text { от } 96 \text { до } 100 \\
98,0 \pm 2,0 \\
\text { от } 44 \text { до } 82 \\
53,7 \pm 18,2 \\
\end{array}$ & $\begin{array}{l}0 \\
0\end{array}$ \\
\hline IV & $\begin{array}{l}\text { Грудной } \\
\text { Грудопоясничный }\end{array}$ & $\begin{array}{c}\text { от } 18 \text { до } 100 \\
63,8 \pm 18,7 \\
\text { от } 46 \text { до } 73 \\
59,0 \pm 12,0\end{array}$ & $\begin{array}{l}0 \\
0\end{array}$ \\
\hline
\end{tabular}

Примечания: в таблице представлены выборочные предельные значения, затем среднее \pm стандартное отклонение. 
Таким образом, основное количество больных оперированы в промежуточный и в поздний периоды от момента травмы.

В ходе хирургического лечения у всех пациентов с позвоночно-спинномозговой травмой достигнута полноценная ликвидация стеноза позвоночного канала, а следовательно, декомпрессия спинного мозга и восстановление анатомии позвоночного канала (табл. 4).

У пациентов I группы стеноз позвоночного канала был более грубым по сравнению с остальными группами и составлял в среднем $93 \%$ в грудном отделе, $84 \%$ в области грудопоясничного перехода.

При неврологическом осмотре среди детей всех групп в раннем послеоперационном периоде отмечался регресс неврологического дефицита в виде восстановления двигательных и чувствительных функций (болевая, тактильная) в среднем на 1-2 балла по ASIA с более быстрым восстановлением болевой чувствительности.

У пациентов первой группы после проведения хирургической декомпрессии улучшение неврологических функций (двигательных и чувствительных) отмечено на 1-2-е сутки после оперативного лечения, у пациентов второй группы - на 2-3-и сутки, третьей группы - на 5-7-е сутки, четвертой - через 4-5 месяцев. Более быстрое восстановление наблюдалось у пациентов первой группы (6 человек), имевших неврологические нарушения типа B по шкале ASIA и прооперированных в первые 6-12 часов от момента травмы.

При оценке отдаленных результатов у обследованных пациентов выявлены следующие результаты.

Групnа 1. У 6 больных с неполным нарушением проведения спинного мозга отмечалась выраженная положительная динамика в виде значительного регресса чувствительных нарушений: у 3 больных отмечено их восстановление до нормы (тип Е), еще у трех уровень болевой и тактильной чувствительности повысился в среднем на 18 баллов от исходных значений. В двигательной сфере у этих пациентов прирост показателей составил в среднем 26 баллов от данных первичного неврологического осмотра. В отдаленном периоде (5 лет) после хирургического лечения у детей этой группы двигательные параметры соответствовали 71 баллу (от 50 до 100), чувствительные - 85 баллам (от 54 до 112); четверо пациентов начали передвигаться самостоятельно, двое - с использованием вспомогательных аппаратов. У двух больных с исходным уровнем неврологических нарушений, соответствующих типу А, изменений со стороны клинической картины не отмечено. У них сохранились нижняя параплегия и нарушения функции тазовых органов.
Группа 2. Полный регресс неврологических расстройств отмечен у двоих пациентов с исходным уровнем нарушений D по шкале ASIA. Еще в двух наблюдениях при исходном типе неврологических нарушений A по шкале ASIA регресса неврологической симптоматики не отмечалось. В отдаленном периоде позвоночно-спинномозговой травмы средние значения двигательных функций соответствовали 73 баллам (от 50 до 97), чувствительных - 95 баллам (от 78 до 112).

Групnа 3. У четырех из 8 пациентов отмечалась положительная динамика с переходом уровня неврологического дефицита с типа С в тип D, y двух - c типа В в тип D. У 2 детей с типом неврологических расстройств $\mathrm{A}$ их регресса не наблюдалось. В отдаленном периоде после хирургического лечения двигательные функции в среднем оценивались в 77 баллов (от 50 до 93), чувствительные - в 91 балл (от 38 до 107). Прирост показателей чувствительности в среднем составил 10 (от 6 до 15) баллов, моторных 19 (от 0 до 34) баллов. К 4-5-му году наблюдения динамика восстановления неврологических нарушений полностью прекращалась.

Грynna 4. У пациентов этой группы с неполным нарушением функции спинного мозга также отмечалось улучшение неврологического статуса после оперативного лечения, но более медленным темпом. Из 16 пациентов этой группы у шестерых детей, имевших изначально тип неврологических нарушений В по шкале ASIA, ко 2-3-му году наблюдений отмечался их регресс до типа С. У 10 больных с уровнем неврологического дефицита А по шкале ASIA положительной динамики в неврологическом статусе не отмечалось. Оценка двигательных функций в четвертой группе пациентов соответствовала в среднем 59 баллам (от 50 до 82), чувствительных - 67 баллам (от 24 до 84 ).

Особое внимание стоит уделить 16 пациентам из всех групп, неврологические нарушения у которых исходно оценены как тип A по шкале ASIA. Вне зависимости от сроков оперативного лечения эти пострадавшие способности к произвольным движениям в нижних конечностях не обрели. Динамика чувствительных функций характеризовалась у них медленным восстановлением в отдаленном периоде (в сроки более 2-3 лет после хирургического лечения), но на величину не более 5-8 баллов от исходного уровня.

\section{ОбсужАение}

Согласно полученным результатам исследования установлено, что у пациентов с повреждением 
позвоночника в грудном отделе позвоночника выраженность неврологических нарушений была более тяжелой по сравнению с локализацией перелома в зоне грудопоясничного перехода. На наш взгляд, это прямым образом связано с меньшим размером позвоночного канала в грудном сегменте позвоночного столба по сравнению с грудопоясничным отделом. В результате этого резервные свободные пространства для спинного мозга на этом уровне оказываются малыми и при переломе позвоночника клиническая выраженность неврологических нарушений оказывается значительной по сравнению с нижележащими сегментами.

Установлено, что чем в более ранние сроки проведено хирургическое вмешательство у пациентов с позвоночно-спинномозговой травмой, тем более быстрыми темпами и полноценнее (вплоть до физиологической нормы) происходит восстановление неврологических нарушений. Исключение составляют пациенты с типом неврологического дефицита A по шкале ASIA, у которых восстановления двигательных функций спинного мозга не отмечено ни в одном наблюдении. Однако положительная динамика в восстановлении чувствительной сферы у детей с типом нарушений А, прооперированных в первые часы от момента травмы, в виде увеличения количества баллов по шкале ASIA явно прослеживалась.

У пациентов первой группы стеноз канала до операции был наиболее выраженный среди всех групп больных и составлял в среднем 93,5 $\pm 3,5$ в грудном отделе и $83,7 \pm 6,7$ в зоне грудопоясничного перехода. У этой категории больных отмечались выраженные неврологические нарушения типа A (2) и В (6) по шкале ASIA. Отсутствие динамики неврологических нарушений у пациентов этой группы с типом повреждения А по шкале ASIA, на наш взгляд, было обусловлено уровнем повреждения (грудной отдел), выраженным стенозом позвоночного канала на этом уровне и функциональным перерывом спинного мозга.

У пациентов этой группы с типом неврологического дефицита В динамика регресса неврологических нарушений после хирургического вмешательства была достоверно выше по сравнению с остальными группами исследования. Кроме того, отмечено, что восстановление двигательных и чувствительных функций происходило более быстрыми темпами (на 1-2-е сутки после операции). Такие положительные изменения у детей первой группы объяснялись ранней и адекватной проведенной декомпрессией спинного мозга и его элементов в сочетании со стабилизацией поврежденного позвоночно-двигательного сегмента. На наш взгляд, именно сдавление спинного мозга и его элементов отломками тел позвонков у этой категории пациентов являлось основной причиной неврологических нарушений. Хирургическое вмешательство, проведенное в первые часы от момента травмы, позволило не только ликвидировать причину сдавления спинного мозга и его структур, восстановить анатомию позвоночного канала, но и предотвратить отек, циркуляторные нарушения и развитие вторичных патологических необратимых процессов в нем.

У пациентов второй группы стеноз позвоночного канала после травмы был менее выраженный по сравнению с больными первой группы и составил в среднем 65,0 \pm 22 . Этот факт полностью соответствовал и вариантам неврологических нарушений - тип A (2) и D (2) по шкале ASIA.

У двух пациентов с типом неврологических нарушений А по шкале ASIA отсутствие динамики восстановления двигательных и чувствительных нарушений связано с выраженным стенозом канала (в среднем $87 \%$ ) на уровне грудопоясничного перехода и тяжестью повреждения спинного мозга.

Несмотря на более поздние сроки проведения хирургического вмешательства у этой группы пациентов, полный регресс неврологического дефицита отмечен у двух детей с типом нарушений D по шкале ASIA. Однако темпы его восстановления были более медленные (на 2-3-и сутки после операции) по сравнению с первой группой больных. По нашему мнению, восстановление неврологических нарушений у этих пациентов связано с незначительным дефицитом (тип D) и уровнем повреждения спинного мозга (грудопоясничный переход).

У пациентов третьей группы стеноз позвоночного канала до операции составлял 98,0 $\pm 2,0$ в грудном отделе и $53,7 \pm 18,2$ в зоне грудопоясничного перехода. Величина стеноза канала в зоне грудопоясничного перехода у данного контингента пострадавших была самая маленькая среди всех групп пострадавших. У этой категории больных отмечались следующие типы неврологических нарушений по шкале ASIA - A (2), B (2) и C (4). Подобные варианты дефицита можно объяснить не только уровнем и величиной стеноза позвоночного канала, но и появлением отека и присоединением вторичных патологических процессов в спинном мозге. У пациентов этой группы с типом неврологических нарушений А по шкале ASIA восстановление функции спинного мозга как в чувствительной, так и в двигательной сфере не отмечалось. Позднее хирургическое вмешательство привело к медленному (на 5-7-е сутки после операции) регрессу неврологического дефицита и незначительной ее динамике у пациентов с неврологическими 
нарушениями типа В (2) и С (4). У этих больных отмечались переход с исходного уровня неврологического дефицита до операции в уровень D и полное прекращение положительной динамики восстановления к 4-5-му году от момента операции. Подобные изменения неврологических нарушений, на наш взгляд, были связаны прежде всего с небольшой величиной стеноза позвоночного канала в зоне грудопоясничного перехода (в этой зоне отмечались повреждения типа В и С) сразу после травмы, который был полностью ликвидирован в процессе хирургического вмешательства.

У пациентов четвертой группы стеноз позвоночного канала составил $63,8 \pm 18,7$ в грудном отделе позвоночника и $59,0 \pm 12,0$ в зоне грудопоясничного перехода. У этого контингента пациентов среди всех остальных групп отмечался самый небольшой размер стеноза позвоночного канала. У этих больных, так же как и у пациентов первой группы, отмечались выраженные неврологические нарушения типа А (10) и В (6) по шкале ASIA. Учитывая менее выраженный стеноз позвоночного канала у этой категории больных, можно предположить, что неврологические нарушения формировались не только путем сдавления отломками тел позвонков спинного мозга и его элементов. Основные причины неврологического дефицита в этот период от момента повреждения, с нашей точки зрения, были обусловлены наличием сосудистых нарушений и вторичных патологических процессов, происходящих в спинном мозге. Эти изменения и поздние сроки выполнения хирургического вмешательства не привели к положительной динамике восстановления неврологических нарушений ни у одного пациента с типом неврологических нарушений А по шкале ASIA. По нашему мнению, это связано с тяжестью повреждения самого спинного мозга и необратимыми патофизиологическими изменениями, которые в нем произошли в результате повреждения. Исключение составили 6 пациентов, которые перешли из варианта неврологического дефицита В по шкале ASIA в тип C только ко 2-3-му году после операции.

У пациентов всех групп с вариантом неврологических нарушений типа A по шкале ASIA восстановления двигательной функции не отмечено. Объясняется это тяжестью и выраженностью повреждения спинного мозга. Медленное восстановление в чувствительной сфере у пациентов с полным повреждением спинного мозга (тип А) в отдаленном периоде наблюдения, вероятнее всего, связано с нейропластичностью и резервными возможностями спинного мозга у пациентов детского возраста.

\section{Заключение}

Таким образом, в ходе исследования установлено, что наиболее тяжелые повреждения с точки зрения неврологических нарушений у детей с позвоночно-спинномозговой травмой возникают при локализации переломов в грудном отделе позвоночника. Характер неврологических изменений не только зависит от уровня повреждения позвоночного столба, но и коррелирует с величиной стеноза позвоночного канала. Операция, выполненная в первые часы от момента травмы, приводит к более быстрому и полноценному восстановлению неврологического дефицита. Хирургическое вмешательство обязательно должно сопровождаться полной ликвидацией всех причин, вызывающих сдавление спинного мозга, восстановлением анатомии позвоночного канала и физиологических профилей на уровне поврежденного позвоночно-двигательного сегмента, а также его стабильной фиксацией в отдаленные периоды наблюдения. Своевременная ликвидация сдавления спинного мозга в первые 6-12 часов от момента травмы является залогом успеха в дальнейшем восстановлении функций спинного мозга и регресса неврологических нарушений. Восстановление функций даже одного сегмента спинного мозга значительно улучшает социальную адаптацию и качество жизни пациента, перенесшего позвоночно-спинномозговую травму.

\section{Список митературы}

1. Аганесов А.Г., Месхи К.Т., Николаев А.П., Костив Е.П. Хирургическое лечение осложненной травмы позвоночника в остром периоде // Вестник травматологии и ортопедии им. Н.Н. Приорова. 2003. - № 3. - C. 44-48. [Aganesov AG, Meskhi KT, Nikolaev AP, Kostiv EP. Surgical Treatment of Complicated Spine Injury in Acute Period. Vestnik travmatologii i ortopedii im. N.N. Priorova. 2003;3:44-48. (In Russ).]

2. Виссарионов С.В. Стабильные и нестабильные повреждения грудного и поясничного отделов позвоночника у детей (клиника, диагностика, лечение): пособие для врачей. - СПб., 2010. [Vissarionov SV. Stable and unstable damage to the thoracic and lumbar spine in children (clinical picture, diagnosis, treatment). Saint-Petersburg, 2010. (In Russ).]

3. Виссарионов С.В., Белянчиков С.М. Оперативное лечение детей с осложненными переломами позвонков грудной и поясничной локализации // Травматология и ортопедия России. - 2010. - № 2(56). - С. 48-50. [Vissarionov SV, Bel'anchikov SM. The surgical treatment of children with complicated fractures of thoracic and lumbar vertebrae. Traumatology and orthopedics of Russia. 2010;2(56):48-50. (In Russ).]

4. Виссарионов С.В., Дроздецкий А.П., Кокушин Д.Н., Белянчиков С.М. Оперативное лечение пациентки 
с переломовывихом в грудном отделе позвоночника // Хирургия позвоночника. - 2011. - № 3. - С. 21-25. [Vissarionov SV, Drozdetsky AP, Kokushin DN, Belyanchikov SM. Surgical treatment of a patient with fracture-dislocation in the thoracic spine. Spine surgery. 2011;3:21-25. (In Russ).]

5. Корнилов Н.В., Усиков В.Д. Повреждения позвоночника. Тактика хирургического лечения. - СПб.: MOPCAPAB, 2000. - 231 c. [Kornilov NV, Usikov VD. Povrezhdeniya pozvonochnika. Taktika khirurgicheskogo lecheniya. Saint-Petersburg: MORSARAV; 2000. 231 p. (In Russ).]

6. Asawma T, Satomi K, et al. Management of patients with an incomplete cervical spinal cord injury. Spinal Cord. 1996;34(10):620-625. doi: 10.1038/sc.1996.111.

7. Farmer J, Vaccaro A, Albert TJ, et al. Neurologic deterioration after cervical spinal cord injury. J Spinal Disord. 1998;11(3):192-196.

doi: 10.1097/00002517-199806000-00002.

8. Vaccaro AR, Daugherty RJ, Sheehan TP, et al. Neurologic outcome of early versus late surgery for cervical spinal cord injury. Spine.1997;22(22):2609-2613. doi: 10.1097/00007632-199711150-00006.

9. Mirza SK, Krengel WF, Chapman JR, et al. Early versus delayed surgery for acute cervical spinal cord injury. Clin. Orthop. 1999;359:104-114.

doi: 10.1097/00003086-199902000-00011.

10. Wagner FC, Chehrati B. Early decompression and neurological outcome in acute cervical spinal cord injuries. J Neurosurg. 1982;56:699-705.

doi: $10.3171 /$ jns.1982.56.5.0699.
11. Fehlings MG, Sekhton LH, Tator C. The role and timing of decompression in acute spinal cord injury: what do we know? What should we do? Spine. 2001;26(24):101-110. doi: 10.1097/00007632-200112151-00017.

12. Bohlman HH, Anderson PA. Anterior decompression and arthrodesis of the cervical spine: long-term motor improvement. Part I. Improvement in incomplete traumatic quadriparesis. J Bone Jt Surg. 1992;74(5):671-682.

13. Carlson GD, Minato Y, Okava A, et al. Early timedependent decompression for spinal cord injury: vascular mechanisms of recovery. J Neurotrauma. 1997:14(12):951-962. doi: 10.1089/neu.1997.14.951.

14. Коновалов А.Н., Лихтерман Л.Б., Потапов А.А. Нейротравматология. - М., 1994. - 356 с. [Коnovalov AN, Likhterman LB, Potapov AA. Neirotravmatologiya. Moscow; 1994. 356 p. (In Russ).]

15. Rosenbluth J. Pathology of demyelinated and dysmyelinated axons. The Axon Structure, Function and Pathophysiology. SG Waxman, JD Kocsis, and PK Stys, editors. Oxford University Press, New York, NY. 1995.

16. American Spinal Injury Association and International Medical Society of Paraplegia, eds. Reference manual of the international standards for neurological classification of spinal cord injury. Chicago, IL: American Spinal Injury Association; 2003.

17. Magerl FP, Aebi M, Gertzbein SD, et al. A Comprehensive classification of thoracic and lumbar injures. Eur. Spine J. 1994;3(4):184-201.

doi: $10.1007 / \mathrm{bf} 02221591$.

\title{
ANALYSIS OF THE INFLUENCE OF VARIOUS FACTORS ON THE COURSE OF NEUROLOGICAL DISORDERS IN CHILDREN WITH SPINAL CORD INJURY
}

\author{
Baindurashvili A.G., Solokhina I.Y., Kokushin D.N., Belyanchikov S.M.
}

The Turner Scientific and Research Institute for Children's Orthopedics, Saint-Petersburg, Russian Federation

Background. The study of the influence of various factors on the course of recovery of neurological disorders in children with spinal cord injuries is an important and relevant problem. The main causes of thoracic and lumbar injuries of the spine in children are road accidents and catatraumas. Anatomical and physiological features of the spine and spinal cord in children have a significant influence on the nature of spinal cord injury, clinical manifestations of the injury, and method of treatment. The degree of spinal canal deformity at the level of the damaged segment is directly proportional to the severity of the neurological disorder. The time between injury to when surgery is performed will strongly influence the nature and course of recovery of motor functions.

Aim. To assess the influence of different factors in pediatric patients with complicated injuries of the spine at the thoracic and thoracolumbar levels on the recovery of neurological disorders.

Materials and methods. The analysis of results of the surgical treatment of 36 children ( 24 boys and 12 girls) aged 3-17 years with damage to the spine and spinal cord in the thoracic spine and thoracolumbar junction, accompanied with neurological deficit in the form of central or peripheral paresis and paralysis, was performed. All patients underwent surgical intervention depending on the type and extent of damage. Clinical methods (i.e., detailed neurological examination) as well as X-ray, CT, and MRI were used as diagnostic methods.

Results. The study revealed that the most severe damage concerning neurological disorders in children with spinal cord injury occurs in the thoracic spine. The extent of neurological changes depends not only on the 
level of damage to the spinal column but also on the magnitude of spinal canal stenosis. Surgery performed in the first hours of the injury leads to a more rapid and full recovery of the neurological deficit.

Conclusion. Therefore, this study found that several factors influence the recovery of neurological disorders in children with spinal cord injury: timing of surgery, localization of the injury, spinal stenosis, the nature of lesions of the spinal cord, and the elements involved.

Keywords: spinal trauma and spinal cord trauma in children, spinal cord injury, ASIA scale, spinal surgery.
Баиндурашвили Алексей Георгиевич - д. м. н., профессор, чл.-корр. РАН, заслуженный врач РФ, директор ФГБУ «НИДОИ им. Г.И. Турнера» Минздрава России. E-mail: turner01@mail.ru.

Солохина Ирина Юрьевна - врач-невролог, научный сотрудник отделения патологии позвоночника и нейрохирургии ФГБУ «НИДОИ им. Г.И. Турнера» Минздрава России.

Кокушин Дмитрий Николаевич - научный сотрудник отделения патологии позвоночника и нейрохирургии ФГБУ «НИДОИ им. Г.И. Турнера» Минздрава России.

Белянчиков Сергей Михайлович - к. м. н., заведующий отделением патологии позвоночника и нейрохирургии ФГБУ «НИДОИ им. Г.И. Турнера» Минздрава России. E-mail: belijanchikov@list.ru.
Baindurashvili Alexei Georgievich - MD, $\mathrm{PhD}$, professor, corresponding member of RAS, honored doctor of the Russian Federation, Director of The Turner Scientific and Research Institute for Children's Orthopedics. E-mail: turner01@mail.ru.

Solokhina Irina Yurievna - MD, neurologist, research associate of the department of spinal pathology and neurosurgery. The Turner Scientific and Research Institute for Children's Orthopedics.

Kokushin Dmitriy Nikolaevich - MD, research associate of the department of spinal pathology and neurosurgery. The Turner Scientific and Research Institute for Children's Orthopedics. Tel.: 8(812)465-28-57.

Belyanchikov Sergei Mikhailovich - MD, $\mathrm{PhD}$, chief of the department of spine pathology and neurosurgery. The Turner Scientific and Research Institute for Children's Orthopedics. E-mail: belijanchikov@list.ru. 